\title{
A COMPARSION OF ACOUSTIC AND PSYCHOACOUSTIC MEASUREMENTS OF PASS-THROUGH HEARING PROTECTION DEVICES
}

\author{
Douglas S. Brungart \& Brian W. Hobbs
}

\author{
Air Force Research Laboratory (AFRL/HECB) \\ 2610 Seventh St., WPAFB, OH 45433 \\ douglas.brungart@wpafb.af.mil \\ brian.hobbs@wpafb.af.mil
}

\author{
James T. Hamil
}

\author{
General Dynamics \\ 5200 Springfield Pike, Dayton, OH 45431 \\ james.hamil@wpafb.af.mil
}

\begin{abstract}
In environments where listeners need to detect low-level sounds while being protected from high-level noises, electronic pass-through hearing protectors (EPHPs) offer an appealing alternative to traditional passive earplugs or earmuffs. In this paper, we compare acoustic measurements of the Head-Related Transfer Functions associated with eight different EPHPs to localization results measured on human listeners with the same devices. The results are discussed in terms of the insights they can provide for the design of improved EPHP systems.
\end{abstract}

\section{INTRODUCTION}

Many hazardous environments involve a combination of quiet sounds that may signal emerging threats in the surrounding area and loud sounds that may cause long-term hearing damage. For example, dismounted soldiers rely on low-level sounds to detect enemy activities, but also require protection from loud sounds that may occur due to weapons fire or nearby explosions.

Until recently, listeners in such environments had to choose either to 1) wear a hearing protection device and risk missing a critical low-level ambient sound, or 2) go without hearing protection and risk temporary or permanent hearing loss. However, within the last few years sophisticated "Electronic Pass-through Hearing Protectors" (EPHPs) have emerged to directly address this problem [1]. These devices differ in design, but all are comprised of the same four primary components: 1) a traditional passive earplug or earmuff hearing protector; 2) external microphones for picking up ambient sounds; 3) internal speakers for presenting these sounds to the ears; and 4) an electronic processing unit designed to pass low-level ambient sounds and block high-level ambient sounds. EPHPs can also provide additional benefits, such as the amplification of very low-level ambient sounds (to allow easier detection of enemy movements) and the ability to directly couple the output of a radio to the listener's ears (to allow more effective communication in noisy environments). However, relatively little quantitative information is available on the performance of these devices, and at the present time no validated design guidelines exist for such factors as microphone placement and input-output gain curves for EPHPs.

Although many aspects of EPHP design are application specific, one common design objective for all EPHP systems is to preserve the ability to localize ambient sounds. Simply put, the user of an ideal EPHP system should be able to localize sounds at least as accurately with the system as they would be able to without it. In
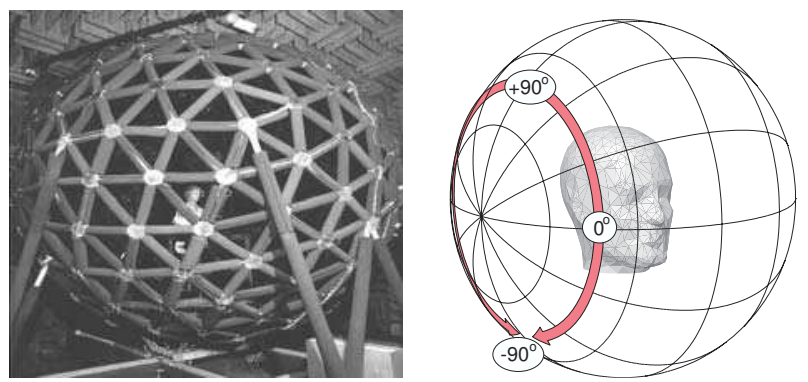

Figure 1: The left panel shows the Auditory Localization Facility $(A L F)$. The right panel shows the interaural-polar coordinate system for the $45^{\circ}$ "cone of confusion" used to plot the HRTFs in Figure 2.

this paper, we present the results of a study that compared localization performance across eight commercially-available EPHPs. We also present the results of acoustical measurements that show how well each device preserved the auditory cues that mediate auditory localization performance, including interaural time delays, interaural level differences, and direction-dependent high-frequency spectral cues. The results are discussed in terms of their implications for the design of improved future EPHPs.

\section{ACOUSTIC MEASUREMENTS}

Devices: Eight EPHPs were tested in the experiment. Four of the systems involved earpieces that were inserted directly into the listener's ears using either a foam or flanged plug ("earplug systems"), and four of the systems involved larger earpieces that fit over the ears and were connected by a spring-loaded headband ("headphone systems"). Although all the systems are now commercially available, some of the measurements reported here used pre-production prototypes rather than production systems. Thus, we have decided to identify the systems only by device type and number. The earplug systems are referred to as P1, P2, P3, and P4, and the headphone systems are referred to as $\mathrm{H} 1, \mathrm{H} 2, \mathrm{H} 3$, and $\mathrm{H} 4$. In the localization portion of the experiment, an additional device called the Combat Arms (CA) earplug (Aearo) was included in the experiment. The CA is a passive earplug that contains a specially designed acoustic filter that passes through low-level sounds but blocks very loud impulsive sounds. Functionally, its purpose is similar to that of an EPHP, and it was therefore included in this study as a point of comparison. 
Apparatus: The acoustic measurements were made with a Knowles Electronics Mankin for Acoustic Research (KEMAR) that was equipped with GRAS IEC 711 Ear Simulators and positioned with its head directly in the center of the Auditory Localization Facility (ALF). The ALF (Figure 1) consists of a large geodesic sphere $(4.3 \mathrm{~m}$ in diameter) suspended in an anechoic chamber to reduce reverberation and ambient noise levels. There are 277 Bose 11-cm full-range loudspeakers mounted at each of the structural vertices of the sphere, with an angular spacing of approximately $15^{\circ}$ between each two speaker locations.

Procedure: The acoustic transfer-function measurements were made using a series of five 2048-point periodic-chirp signals covering the frequency range from $100 \mathrm{~Hz}$ to $15 \mathrm{kHz}$. The signals were generated in MATLAB, and the resulting left- and right-ear microphone signals were divided by the test signals in the frequency domain to provide an estimate of the total system response (i.e. speaker, microphone, propagation path, and Head-Related Transfer Function (HRTF)) from the active loudspeaker location to each of the manikin's ears.

Two sets of measurements were made on each EPHP device. The first was an input-output gain-curve measurement. For the purposes of this measurement, the test signal was simultaneously played through the 15 speakers closest to the front of the sphere in order to generate a combined signal at the center of the sphere that ranged from $42 \mathrm{~dB}$ to $114 \mathrm{~dB}$ SPL in $6 \mathrm{~dB}$ steps ${ }^{1}$. The average RMS levels generated at the left and right ears with the EPHP were then compared to those measured without the EPHP in order to construct an overall input/output gain curve for the device.

The second measurement made on each device was an HRTF measurement where a test signal with an RMS level of $65 \mathrm{~dB}$ SPL was used to measure the left- and right- ear transfer functions for sounds originating from each of the 277 speakers in the ALF. These measurements were then corrected for the individual responses of each loudspeaker to produce an overall estimate of the HRTF for each location.

\subsection{Results}

The results of the acoustic measurements are shown in Figure 2. Each column of the figure shows a different parameter from the measurements, and each row shows a different EPHP (as indicated by the label in the upper left of each panel in the first column). For comparison, the first row shows the result of each measurement in the "open ear" KEMAR condition with no EPHP. Each column is described in more detail below:

Input/Output Gain Curve: The first column shows the mean RMS output level at each ear as a function of the RMS level of the input test signal at the location of the center of the head. In this study, the volume controls of each EPHP were adjusted to produce unity gain when the test signal level was $65 \mathrm{~dB}$ SPL. Thus, the gain curves shown in the leftmost column of Figure 2 all show an output level of $65 \mathrm{~dB}$ SPL for an input level of $65 \mathrm{~dB}$ SPL. Note that most of the devices had a linear response in the output range around $65 \mathrm{~dB}$, but that $\mathrm{P} 2, \mathrm{H} 3$, and $\mathrm{H} 4$ had gain curves with a slope less than one, indicating that the signal was electronically compressed in that region.

Overall Mean Frequency Response: These curves show the mean magnitude of the HRTF averaged across the left and right ears at all of the 277 possible speaker locations. The devices varied

\footnotetext{
${ }^{1} 15$ speakers were required to obtain undistorted output at $114 \mathrm{~dB}$.
}

widely in bandwidth, but all of the devices exhibited substantially more high-frequency roll-off than the open-ear condition.

Interaural Time Delay: The third column shows Interaural Time Delay (ITD) as a function of azimuth location for each EPHP. These values were generated from the best linear fit to the interaural phase difference in the frequency range from 500 to $1500 \mathrm{~Hz}$. The four earplug systems exhibited ITD values that were nearly identical to the open-ear condition, while the four headphone systems produced ITD curves that were similar to one another but had slightly sharper peaks at $\pm 90^{\circ}$ than in the open-ear condition.

Interaural Level Difference: The fouth column shows Interaural Level Difference (ILD) as a function of azimuth angle for each EPHP. These values were generated by averaging the interaural magnitude differences in the HRTFs across three frequency ranges $(1.5-3 \mathrm{kHz}, 3-6 \mathrm{kHz}$, and $6-12 \mathrm{kHz})$. In order to remove any overall level biases across the two ears that might occur due to differences in fit, the ILD values were calculated independently for the left-ear and right-ear HRTFs (with the assumption of leftright symmetry) and averaged together to give the values shown in Figure 2. With the exceptions of P3 and P4, most of the EPHPs produced some compression in the ILD values at high frequencies, which ranged from $+20 \mathrm{~dB}$ to $-20 \mathrm{~dB}$ in the open-ear conditions but only from $+10 \mathrm{~dB}$ to $-10 \mathrm{~dB}$ for most of the devices tested. Devices H3 and H4 showed the most compression at low frequencies, and device $\mathrm{H} 3$ actually produced "inverse" ILD values (i.e., a signal louder at the contralateral ear than the ipsilateral ear) at frequencies less than $3 \mathrm{kHz}$.

Head-Related Transfer Function: The color images in the rightmost column show how the magnitude spectrum of the rightear HRTF changed as a function of vertical angle within the $45^{\circ}$ "cone of confusion" illustrated in the right panel of Figure 1. Thus, a vertical angle of $0^{\circ}$ indicates the point in the horizontal plane $45^{\circ}$ to the right of the listener's midline. Localization in elevation is believed to be mediated by the complex patterns of elevationdependent peaks and notches that are clearly visible in the openear HRTF plot shown in the top-right panel of Figure 2. Subjectively, it does not appear that any of the devices did a very good job of preserving this pattern. Indeed, most of the devices produced HRTF patterns resembling "vertical stripes," suggesting that elevation had little or no impact on the frequency responses of these systems (and thus indicating that elevation localization performance is likely to be poor with these devices).

\section{LOCALIZATION TESTING}

\subsection{Methods}

The localization experiments were conducted by listeners standing in the center of the ALF while fitted with the appropriate EPHP. Eight experienced paid volunteer subjects with normal hearing (4 male, 4 female, age range 20-25 years) participated in the study. The listeners wore a orientation tracking device on a headband and wielded a hand-held tracking device (Intersense MiniTrax) that allowed them to move an LED cursor to the perceived location of the sound source and press a button to indicate a localization response.

The experiment used pink noise stimuli that were presented in two durations, a $250 \mathrm{~ms}$ 'burst' and a 'continuous' noise that remained on until the listener made the localization response. Before each presentation, the signal was convolved with the inverse transfer function of the presentation loudspeaker to eliminate spectral differences across loudspeakers. All the stimuli were presented at an RMS level of $65 \mathrm{~dB}$ SPL at the location of the listener's head. 

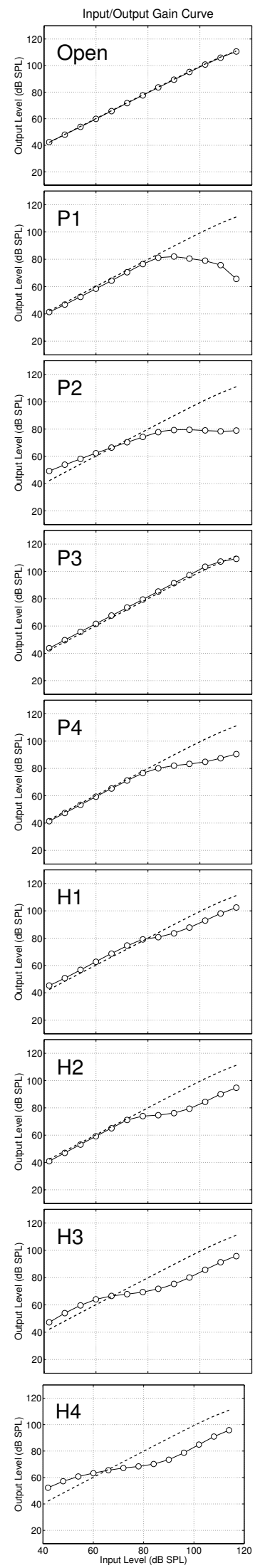
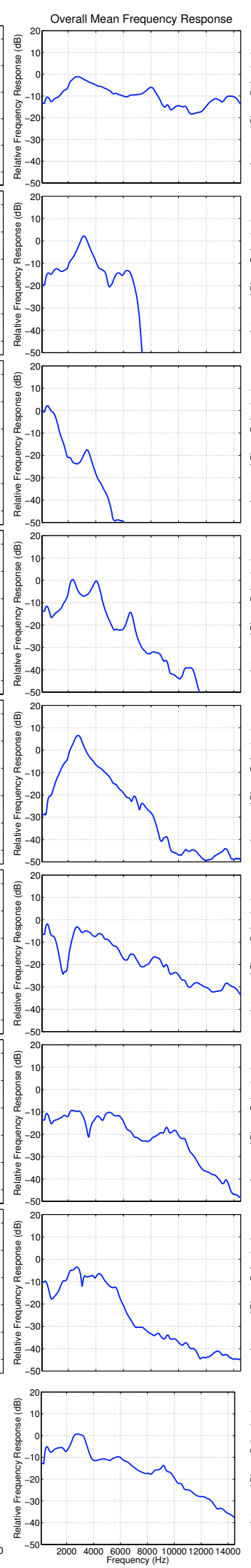
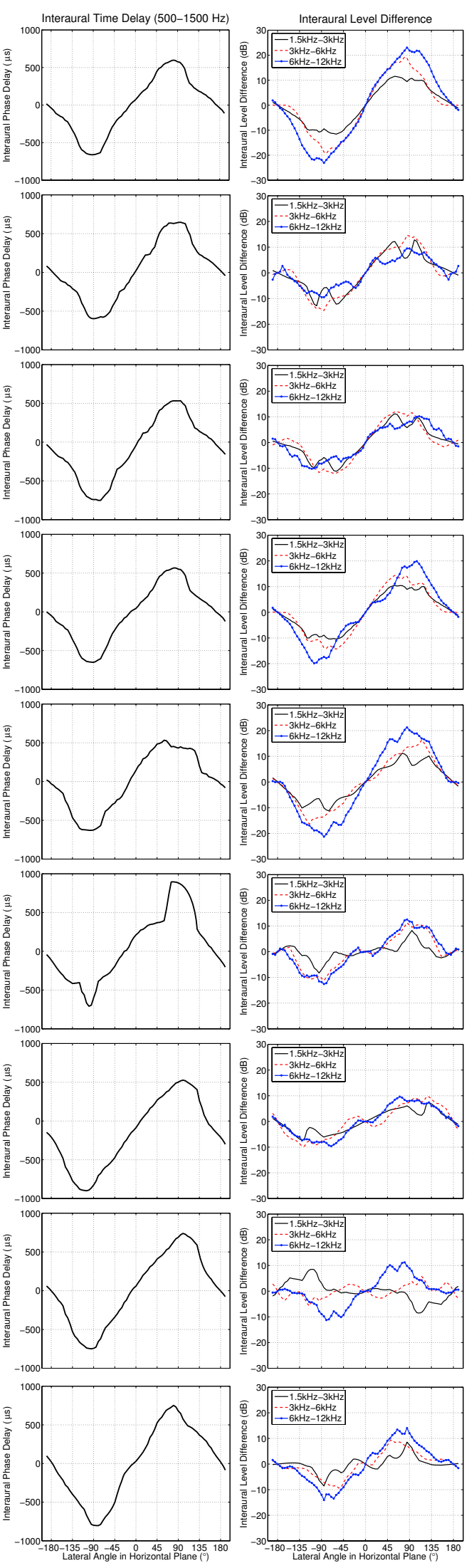
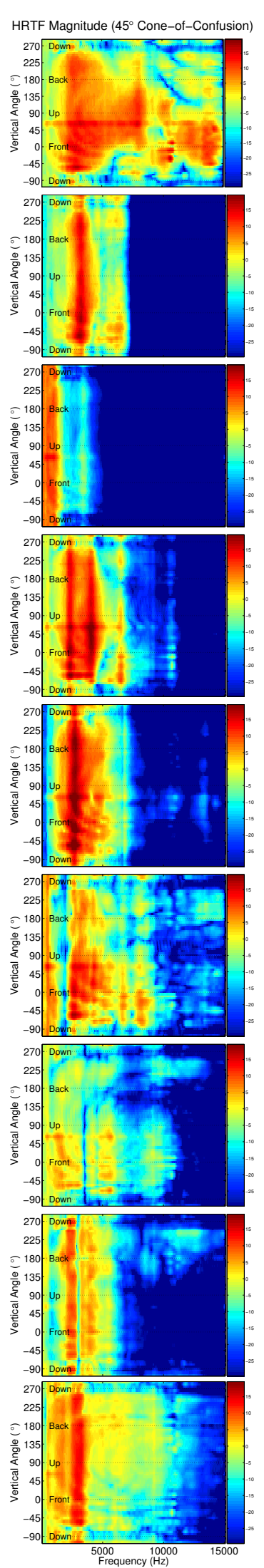

Figure 2: Acoustic measurements made on a KEMAR manikin fitted each of the eight EPHPs. For comparison, the first row shows identical measurements made on a KEMAR manikin with open ear canals. 

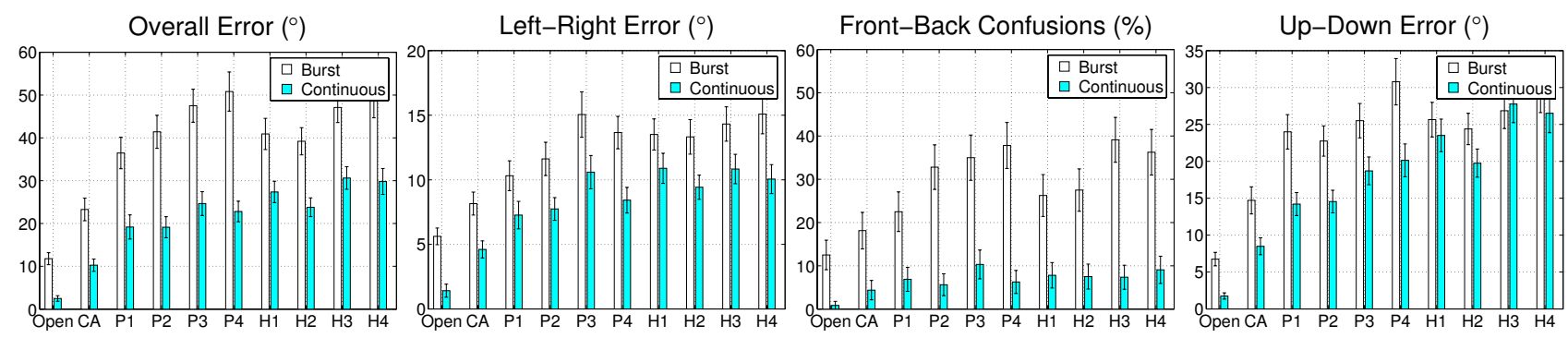

Figure 3: Localization results for each EPHP. The error bars represent $95 \%$ confidence intervals for each data point.

At the start of each trial, the orientation of the listener's head was measured with the headtracking device and a pink noise stimulus was generated from a random speaker location in the sphere with an elevation value greater than $-45^{\circ}$. Then the listener was asked to identify the location with the moveable LED cursor. Correct answer feedback was provided by turning on an LED cluster at the actual source location. The localization responses were collected in two blocks of 180 trials for each device, with a short break after 90 trials within each block. A total of 320 burst and 40 continuous trials were collected for each listener in each EPHP condition.

\subsection{Results and Conclusions}

The results of the localization experiment are shown in Figure 3. The leftmost panel shows the mean "great circle" angular localization error for each EPHP. The second panel shows the mean overall azimuth error in the "left-right" dimension (i.e., with both the stimulus and response locations projected into the front hemisphere). The third panel shows the percentage of "front-back confusions," where the stimulus was in the front hemisphere and the response was in the rear hemisphere or vice versa. Finally, the fourth panel shows the mean absolute angular error in elevation. The data are complicated, but there are number of important conclusions that can be drawn by comparing the localization results shown in Figure 3 to the acoustic measurements shown in Figure 2.

1. Overall localization performance was much poorer for the EPHPs than it was for either the open-ear condition or the passive CA earplug condition. In the open-ear condition, the overall angular error was $12^{\circ}$ in the burst condition and only $2-3^{\circ}$ in the continuous condition, indicating that listeners in that condition could almost always identify the correct target loudspeaker location. In the CA condition, the errors were roughly $22^{\circ}$ in the burst condition and $10^{\circ}$ in the continuous condition. For the EPHPs, burst errors ranged from $35^{\circ}$ to $50^{\circ}$ and continuous errors ranged from $18^{\circ}$ to $30^{\circ}$. Thus, one can argue that none of the EPHPs tested came close to achieving the goal of preserving openear localization accuracy. This is perhaps not surprising considering that none of the HRTF patterns shown in Figure 2 accurately reproduced the direction-dependent frequency response characteristics contained in the open-ear HRTF.

2. Despite considerable differences in microphone placement, compression algorithm, etc., localization performance was comparable across all the EPHPs tested. Some devices performed better than others, but there was no single device that was unambiguously better or worse than the others in any category.

3. System bandwidth is a poor predictor of localization accuracy. The P2 device had, by far, the most restricted bandwidth, sharply limited to only about $4-5 \mathrm{kHz}$. However, it performed no worse than the $\mathrm{P} 1$ device and better than the $\mathrm{P} 3$ and $\mathrm{P} 4$ devices with much broader frequency responses. The $\mathrm{H} 4$ device had one of the widest bandwidths, but was one of the worst performers in almost every category. This suggests that it is not sufficient to simply preserve high frequency content in order to enable localization. Rather, it is necessary to preserve the correct direction-dependent high frequency content in order to maintain a high level of localization accuracy.

4. The preservation of ITD and ILD is not sufficient to ensure good left-right localization performance. Left-right localization performance was impaired relative to the open-ear condition for all the EPHPs, especially in the continuous condition, despite the reasonably accurate mean ITD and ILD values shown in the third and fourth columns of Figure 2. This may have occurred as a result of the introduction of interaural level biases due to variations in the fit of the devices on the listener's two ears.

5. The introduction of a directional gain towards the front of the listener can help prevent front-back confusions. The results in the third panel of Figure 3 show a substantially smaller number of front-back confusions for devices $\mathrm{P} 1, \mathrm{H} 1$, and $\mathrm{H} 2$ in the burst condition than for the other devices. This seems to correlate with the HRTF measurements in Figure 2, which show that these devices have more high-frequency content for sources in front of the listener (bottom half of the panels) than sources behind the listener. This appears to be the one of the few aspects of the localization results that can be explained directly from the acoustic measurements shown in Figure 2.

Overall, these results suggest that, while the current generation of EPHPs clearly represents an improvement in functionality over passive hearing protections, much work still needs to be done to develop an EPHP system that meets the goal of providing truly transparent hearing for ambient sounds. Certainly one mystery is why the passive Combat Arms earplug, which fills much of the concha and attenuates some high-frequency sound energy, performed so much better than the EPHPs in almost every category. It is also worth noting that the EPHP results presented here are much worse than those obtained in a previous study from our laboratory that used similar methods to evaluate localization for a set of ana$\log$ EPHPs with a variety of different microphone configurations [1]. That study reported similar localization results in the open-ear condition, but overall angular EPHP errors of only about $10^{\circ}$ for continuous sounds and $25-35^{\circ}$ for sound bursts. At this point, it is not clear why those devices performed so much better than this current group of EPHPs. Clearly more research is needed to fully understand all of the factors that influence localization accuracy in the design of EPHPs.

\section{REFERENCES}

[1] Brungart, D. S., Kordik, A. J., Eades, C. S., Simpson, B. D., "The effect of microphone placement on localization accuracy with electronic pass-through earplugs," in Proceedings of 2003 IEEE Workshop on Applications of Signal Processing to Audio and Acoustics, New Paltz, New York, Oct 19-22, 2003. 